New Theory and Methods in Stochastic Mixed Integer Programming

Oleg Prokopyev

UNIVERSITY OF PITTSBURGH

08/04/2014

Final Report

DISTRIBUTION A: Distribution approved for public release.

Air Force Research Laboratory AF Office Of Scientific Research (AFOSR)/ RTA

Arlington, Virginia 22203

Air Force Materiel Command 


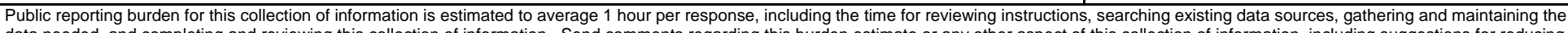

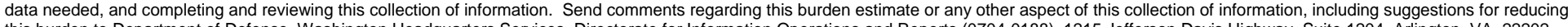

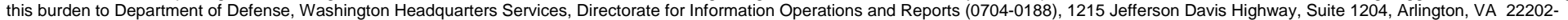

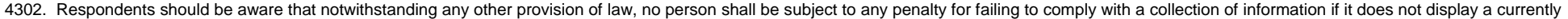
valid OMB control number. PLEASE DO NOT RETURN YOUR FORM TO THE ABOVE ADDRESS.

\begin{tabular}{l|l|l} 
1. REPORT DATE (DD-MM-YYYY) & 2. REPORT TYPE & 3. DATES COVERED (FrOm - To)
\end{tabular}

$07 / 11 / 2014$

Final

May 2011 - Apr2014

4. TITLE AND SUBTITLE

New Theory and Methods in Stochastic Mixed Integer Programming

5a. CONTRACT NUMBER

5b. GRANT NUMBER

FA9550-11-1-0037

5c. PROGRAM ELEMENT NUMBER

6. AUTHOR(S)

Prokopyev, Oleg, A.

5d. PROJECT NUMBER

5e. TASK NUMBER

5f. WORK UNIT NUMBER

7. PERFORMING ORGANIZATION NAME(S) AND ADDRESS(ES)

University of Pittsburgh

8. PERFORMING ORGANIZATION REPORT NUMBER

Office of Research

123 University Place

Pittsburgh, PA 15213-2303

9. SPONSORING I MONITORING AGENCY NAME(S) AND ADDRESS(ES)

10. SPONSOR/MONITOR'S ACRONYM(S)

Air Force Office of Scientific Research

875 N. Randolph Street, Room 3112

Arlington, VA 22203

11. SPONSOR/MONITOR'S REPORT NUMBER(S)

\section{DISTRIBUTION I AVAILABILITY STATEMENT}

Distribution unlimited

\section{SUPPLEMENTARY NOTES}

\section{ABSTRACT}

The proposed project was aimed at exploring various theoretical and algorithmic issues at the intersection of three optimization areas, namely, parametric, stochastic and bilevel integer programming, as well as related applications. The main contribution of the project is development of novel algorithmic methodologies (along with the necessary theoretical foundations) for solving stochastic and bilevel integer programs built upon exploiting equivalent value function reformulations. While computational limitations exist for the proposed approaches, the preliminary results of our experiments are extremely encouraging as for several broad classes of stochastic and bilevel integer optimization problems we are able to solve instances that are among the largest instances solved in the literature. Additionally, we explore several interesting related applications including those arising in wireless sensor networks (and, possibly, other networked systems). For the considered applications we derive structural properties of the optimal policies and exploit them to develop exact solution techniques. Finally, we provide theoretical investigation of randomized restart algorithms in the context of algorithm portfolios.

\section{SUBJECT TERMS}

stochastic integer programming; bilevel integer programming; analysis of algorithms; branch-and-bound; heuristics; value functio

\begin{tabular}{|l|l|l|l|l|l|}
\hline \multicolumn{2}{|l|}{ 16. SECURITY CLASSIFICATION OF: } & $\begin{array}{l}\text { 17. LIMITATION } \\
\text { OF ABSTRACT }\end{array}$ & $\begin{array}{l}\text { 18. NUMBER } \\
\text { OF PAGES }\end{array}$ & $\begin{array}{l}\text { 19a. NAME OF RESPONSIBLE PERSON } \\
\text { Allen A. DiPalma }\end{array}$ \\
\cline { 1 - 2 } $\begin{array}{l}\text { a. REPORT } \\
\text { Unclassified }\end{array}$ & b. ABSTRACT & c. THIS PAGE & UU & 6 & $\begin{array}{l}\text { 19b. TELEPHONE NUMBER (include area } \\
\text { code) } \\
412-624-7400\end{array}$ \\
\end{tabular}




\title{
New Theory and Methods in Stochastic Mixed Integer Programming
}

\author{
PI: Oleg A. Prokopyev \\ Department of Industrial Engineering, University of Pittsburgh \\ 1048 Benedum Hall, Pittsburgh, PA 15261 \\ Phone: 412-624-9833, Fax: 412-624-9831 \\ E-mail: prokopyev@engr.pitt.edu
}

Grant \#: FA9550-11-1-0037

Final Project Report

$05 / 01 / 2011-4 / 30 / 2014$

Program Manager: Dr. Fariba Fahroo

E-mail: fariba.fahroo@us.af.mil

\begin{abstract}
The proposed project was aimed at exploring various theoretical and algorithmic issues at the intersection of three optimization areas, namely, parametric, stochastic and bilevel integer programming, as well as related applications. The main contribution of the project is development of novel algorithmic methodologies (along with the necessary theoretical foundations) for solving stochastic and bilevel integer programs built upon exploiting equivalent value function reformulations. While computational limitations exist for the proposed approaches, the preliminary results of our experiments are extremely encouraging as for several broad classes of stochastic and bilevel integer optimization problems we are able to solve instances that are among the largest instances solved in the literature. Additionally, we explore several interesting related applications including those arising in wireless sensor networks (and, possibly, other networked systems). For the considered applications we derive structural properties of the optimal policies and exploit them to develop exact solution techniques. Finally, we provide theoretical investigation of randomized restart algorithms in the context of algorithm portfolios (i.e., set of algorithms run in parallel). In particular, we provide the theoretical upper bound on the computational value of mixing randomized restart algorithms with different properties. Furthermore, the constructive proof of the main result allows us to characterize restart algorithms that are capable of forming an effective mixed algorithm portfolio.
\end{abstract}




\section{Summary of Research Contributions}

The proposed project was aimed at exploring various theoretical and algorithmic issues at the intersection of three optimization areas, namely, parametric, stochastic and bilevel integer programming, as well as related applications. Below, we briefly summarize the main results and contributions of the project, which are organized in three sections. Specifically, we first consider our work (both theoretical and algorithmic) concerned with novel solution approaches (mostly focusing on parametric programming based) for stochastic integer and mixed integer programs (stochastic IPs and MIPs, respectively) $[6,12,13]$ as well as bilevel IPs [1, 2]. Second, we discuss several applications studied in the framework of the project, in particular, some classes of stochastic assignment, stochastic knapsack and maintenance optimization problems $[3,4,5,11]$. Finally, we briefly describe some additional related work including our results on randomized restarts algorithms [7] and two other publications [14, 15], which, while not being directly related to the goals of the project, resulted from the collaboration with AFRL researchers facilitated by this grant.

\subsection{Parametric programming for stochastic and bilevel optimization problems}

\subsubsection{Solving stochastic IPs and MIPs}

In [13], we examine the value function of pure IPs with inequality constraints. We show that the value function can be characterized entirely by its value on the set of vectors that are minimal with respect to their objective function level set. Unfortunately, membership in this set is, in general, difficult to verify. Thus, we discuss a superset that maintains many of its desirable properties and yet can be generated in a straightforward manner. More importantly, we demonstrate the usefulness of our characterization in the context of stochastic IPs. Specifically, we consider two-stage pure IPs with stochastic right-hand sides, which can be solved, among other alternatives, using a value function reformulation for both stages. The focus is on a class of solution methods based on the following idea:

- Phase 1: compute and completely store in the computer memory first- and second-stage value functions for all (or almost all) right-hand sides (these sets are assumed to be finite);

- Phase 2: apply a global branch-and-bound $(B \& B)$ algorithm over the set of right-hand sides of the first-stage value function.

As demonstrated in the previous work in the literature (see, e.g., somewhat related PI's work in [9] funded by an earlier grant from AFOSR), one of the main advantages of such approaches is their relative insensitivity to the number of variables and scenarios. However, this comes at the price of sensitivity to both the number of constraints in each stage and the magnitude of right-hand sides because of value function storage requirements. In [13], we improve on such limitations by demonstrating that an optimal solution to the value function reformulation exists over the sets of level-set minimal vectors corresponding to both stages. These sets of level-set minimal vectors are generally much smaller than the entire set of interesting righthand sides, leading to greatly reduced memory needs. Coupled with an efficient value function lookup for any given right-hand side, our approach (global branch-and-bound based) is often able to effectively solve stochastic pure integer programs with a greater number of constraints. While computational limitations exist for our approach, our experiments demonstrate that we 
are able to solve instances of two-stage stochastic IPs with extensive forms that are among the largest instances solved in the literature. Furthermore, our approach might be amenable to solve more general two-stage stochastic integer programs as long as the scenarios can be divided into relatively few groups sharing the same technology and recourse matrices as well as the same second-stage objective.

In [12], we continue studying value function based approaches for solving two-stage stochastic IPs. We attempt to improve on one of the limitations of such methods discussed above, namely, the small magnitude of right-hand sides, which can be handled by the solution methods based on storing exact value functions, and then exploiting this information within a global B\&B framework. The main contribution of [12] is a relatively simple approach to reduce memory requirements for this class of solution methods. In particular, instead of exact value functions we compute and store their approximations (specifically, their upper bounds) constructed using the constraint aggregation idea. The main advantage of the proposed concept is its reduced memory requirements, under some mild conditions. These approximations are subsequently exploited to compute appropriate upper bounds within a global B\&B algorithm. In our computational experiments we use a set of test instances generated based on similar parameters to [13] but with larger right-hand sides. Limited computational experiments with a rather naive implementation of the proposed approach on a class of test instances still compare favorably to other methods in the literature (including [13] and exact commercial solvers). Our treatment leaves a number of considerations for future investigation. Nevertheless, we believe that constraint aggregation ideas may be useful, at least for certain problem classes, in the context of value functions and stochastic integer optimization.

Both of the above papers focus on pure IP case. In [6], we attempt to explore value function based approaches for solving two-stage stochastic mixed IPs (i.e., there are no integrality restrictions for some subsets of the fist- and second-stage variables). Our computational experiments are extremely encouraging. The paper is at the final stage of preparation for submission to a refereed journal.

\subsubsection{Solving bilevel IPs}

Bilevel programs form a class of optimization problems that model the hierarchical relationship between two independent (conflicting or, possibly, collaborating) decision-makers (DMs), namely, the leader (e.g., attacker) and the follower (e.g., defender). The decisions are performed sequentially with the leader (upper-level DM) acting first. The follower (lower-level DM) solves his/her own optimization problem, the parameters of which depend on the leader's decisions. The leader's objective is a function of both the leader's and followers decision variables. Thus, the leader should decide by considering the follower's response referred to as the lower-level reaction set. Applications of bilevel programming are numerous including problems in network design and defense (e.g., attacker-defender or defender-attacker problems).

In [1], we consider a general class of nonlinear bilevel knapsack problems (NBKPs), where the leader controls some resource and decides on the allocation of this resource between himself/herself and the follower. Consequently, both the leader and the follower solve 0-1 knapsack problems, where the right-hand sides of the linear knapsack constraints in both problems depends on the resource allocated by the leader. The follower's objective function is assumed to be linear, while the leader's objective is a sum of two general (possibly nonlinear) functions of the leader's and the follower's decisions, respectively. The contributions of [1] are as follows:

(i) We make two modeling extensions to bilevel programs with knapsack constraints dis- 
cussed in the literature. First, we consider a class of problems, where the leader's objective is in a rather general functional form (admittedly, with some mild assumptions). Second, the capacity of the follower's knapsack constraint depends on the leader's resource allocation decision in a generic functional form. Therefore, NBKP model is capable of capturing situations, where (a) the follower's decisions have a nonlinear effect on the leader's objective, and $(b)$ the follower's available capacity is an arbitrary function of the leader's resource allocation decision (e.g., some of the allocated resources may be lost during the shipment process of the resource).

(ii) We demonstrate that several other classes of BKPs previously considered in the literature are a special case of NBKP. We provide theoretical computational complexity results for BKP establishing that $(a)$ the problem remains difficult ( $N P$-hard and not approximable in polynomial time) even there is an unlimited resource for the leader and $(b)$ the problem of checking whether a given feasible solution is locally optimal (with respect to some simple neighborhood of the leader's integer decision) is also difficult.

(iii) Exploiting an equivalent single-level value function based reformulation, we propose an exact solution approach for solving NBKPs. We also tailor the general method for two special classes of nonlinear objective functions, namely, quadratic and fractional $0-1$ functions. Finally, we provide an extensive computational study demonstrating the performance of the developed algorithms. In some respect, our solution approach can be viewed as a (non-trivial) generalization of our previous approach (see, e.g., somewhat related PI's work in [8] funded by an earlier grant from AFOSR) for the case of nonlinear integer functions. Thus, given a generic functional form (with respect to the leader's decisions) of the follower's available capacity, our approach can be easily tailored to handle uncertainty of right-hand sides similar to [8].

In our most recent related work [2] (performed jointly with a researcher from AFRL) we study a general class of bilevel extensions of the classical assignment problem, where each DM has its own objective function and controls a distinct set of edges in a given bipartite graph. Specifically, we propose an exact solution approach, which is based on a branch-and-bound framework and exploits structural properties of the assignment problem. Extensive computational experiments with linear sum and linear bottleneck objective functions are conducted to demonstrate the performance of the developed methods.

\subsection{Related applications: stochastic knapsack, assignment and maintenance problems}

In our grant we proposed to consider several potential applications, including stochastic assignment and knapsack-like optimization problems. Thus, in [11] we are concerned with a class of chance-constrained knapsack problems, which have a number of possible applications, in particular, in health care (real-time operating room batch scheduling). We develop a novel class of solution approaches based on a normal approximation of uncertain problem parameters and demonstrate its excellent performance on test instances generated using real-life data. In [4], we propose a class of greedy approximation algorithms for two-stage assignment problems generalizing some of the previous work in the literature. Most of the work on [4] was performed under previous funding from AFOSR; however, after submission to a refereed journal some revisions were required, which were performed under the current grant as some of the results from [4] fits its scope. Finally, in [3,5] we consider two classes of maintenance optimization problems, which have applications in wireless sensor networks optimal maintenance under uncertainty. In both papers, we derive structural properties of the optimal policies and exploit them to develop exact solution techniques. 


\subsection{Other Related Work}

As it was mentioned in our grant, for many classes of difficult stochastic optimization problems, application of heuristic approaches is necessary. In [7], we focus on randomized optimization algorithms. The presence of some type of randomization is an intrinsic part of their design, which often results in algorithms that significantly outperform their deterministic counterparts in terms of computational effectiveness. More importantly, by introducing randomness into deterministic methods, one can achieve substantial algorithmic speedup in many practical situations. One interesting example is opportunistic parallel optimization mode in CPLEX solver (perhaps, the best commercial IP and MIP solver). Thus, in [7] we discuss two possible parallel strategies for randomized restart algorithms. Specifically, given a set of available algorithms, one can either choose the best performing algorithm and run its multiple copies in parallel (single algorithm portfolio) or choose some subset of algorithms to run in parallel (mixed algorithm portfolio). It has been previously shown that the latter approach may provide better results computationally. We provide theoretical investigation of the extent of such improvement generalizing some of the known results from the literature (including the PI's earlier work in [10]). In particular, we provide the theoretical upper bound on the computational value of mixing randomized restart algorithms with different properties. Furthermore, the constructive proof of the main result allows us to characterize restart algorithms that are capable of forming an effective mixed algorithm portfolio.

Finally, we should mention two other publications, which, while not being directly related to the goals of the project, resulted from the collaboration with AFRL researchers facilitated by this grant. The first paper [14] is concerned with finding maximum subgraphs with relatively large vertex connectivity. The second paper [15] proposes a novel framework for identifying the most "significant" nodes and edges (referred to as critical nodes and edges, respectively) in networks, specifically, those whose removal (or deletion) maximally degrades the connectivity of the network according to some pre-defined metric. The concept of critical nodes and edges allows for the characterization of vulnerability and robustness properties of a given networked system with respect to node and edge removals, which may be caused by adversarial attacks, random failures due to operating conditions, or natural disasters. Such issues are of great concern in a variety of application domains including telecommunication, transportation, social network analysis, homeland security and defense areas.

\section{References}

[1] B. Beheshti, O.Y. Özaltın, M.H. Zare, and O.A. Prokopyev. Exact solution approach for a class of nonlinear bilevel knapsack problems. Journal of Global Optimization, 2014. accepted for publication.

[2] B. Beheshti, O.A. Prokopyev, and E.L. Pasiliao. Exact solution approach for the bilevel assignment problem. 2014. Technical report (submitted for possible publication in a refereed journal).

[3] H. Kai, L.M. Maillart, and O.A. Prokopyev. Scheduling preventive maintenance as a function of an imperfect inspection interval. 2014. Technical report (submitted for possible publication in a refereed journal). 
[4] S. Karademir, N. Kong, and O.A. Prokopyev. On greedy approximation algorithms for a class of two-stage stochastic assignment problems. Optimization Methods and Software, 29(1):42-67, 2014.

[5] A. Khojandi, L.M. Maillart, and O.A. Prokopyev. Optimal planning of life-depleting maintenance activities. IIE Transactions, 46(7):636-652, 2014.

[6] S.V. Kuznetsov, O.A. Prokopyev, and A.J. Schaefer. A value function approach for solving a class of two-stage stochastic mixed-integer programs. 2014. Working paper.

[7] O. Mostovyi, O.A. Prokopyev, and O.V. Shylo. On maximum speedup ratio of restart algorithm portfolios. INFORMS Journal on Computing, 25(2):222-229, 2013.

[8] O.Y. Özaltın, O.A. Prokopyev, and A.J. Schaefer. The bilevel knapsack problem with stochastic right-hand sides. Operations Research Letters, 38(4):328-333, 2010.

[9] O.Y. Özaltın, O.A. Prokopyev, and A.J. Schaefer. Two-stage quadratic integer programs with stochastic right-hand sides. Mathematical Programming, 133(1-2):121-158, 2012.

[10] O.V. Shylo, O.A. Prokopyev, and J. Rajgopal. On algorithm portfolios and restart strategies. Operations Research Letters, 39(1):49-52, 2011.

[11] O.V. Shylo, O.A. Prokopyev, and A.J. Schaefer. Stochastic operating room scheduling for high-volume specialties under block booking. INFORMS Journal on Computing, 25(4):682692, 2012.

[12] A.C. Trapp and O.A. Prokopyev. On constraint aggregation and value functions for twostage stochastic integer programs. 2014. Technical report (submitted for possible publication in a refereed journal).

[13] A.C. Trapp, O.A. Prokopyev, and A.J. Schaefer. On a level-set characterization of the value function of an integer program and its application to stochastic programming. Operations Research, 61(2):498-511, 2013.

[14] A. Veremyev, O.A. Prokopyev, V. Boginski, and E.L. Pasiliao. Finding maximum subgraphs with relatively large vertex connectivity. European Journal of Operational Research, 239(2):349-362, 2014.

[15] A. Veremyev, O.A. Prokopyev, and E.L. Pasiliao. An integer programming framework for critical elements detection in graphs. Journal of Combinatorial Optimization, 28(1):233-273, 2014. 